\title{
LAS DIMENSIONES DE LA RESPONSABILIDAD SOCIAL DE LAS EMPRESAS: UNA GUÍA DE LECTURA PARA SU ESTUDIO*
}

\author{
RICARDO CUEVAS MORENO" \\ UNIVERSITÉ DU QUÉBEC A MONTRÉAL (CANADÁ)
}

Recibido/ Received/ Recebido: 06/12/2010 - Aceptado/ Accepted / Aprovado: 04/04/2011

\begin{abstract}
Resumen
Este artículo propone una guía de lectura para el estudio de la RSE mediante tres dimensiones (herramienta de la actualidad, utilidad para la administración y construcción ideológica) las cuales corresponden lógica e históricamente a la evolución de la RSE. En efecto, el estado de conocimiento y desarrollo de la RSE en las últimas dos décadas se cristaliza en un discurso y una serie de herramientas en la actualidad. El juego de útiles para su aplicación y evaluación constituye la dimensión de útil para la administración. Al discurso corresponde la dimensión ideológica y doctrinaria; de tal manera que éstas se entrelazan en la praxis de la administración. Estas dimensiones permiten comprender el contenido y la forma de la RSE. En otros términos, esta división teórica tridimensional es al propio tiempo una guía de lectura para el estudio de la RSE. Se concluye, que pese al progreso que la responsabilidad social empresarial representa, subsiste la contradicción entre su vocación humanista/ecologista frente al interés privado.
\end{abstract}

Palabras clave: Responsabilidad social de la empresa, dimensión de útil de la administración, dimensión ideológica, totalidad, práctica organizacional.

\section{DIMENSIONS OF ENTERPRISE SOCIAL RESPONSIBILITY: A READING GUIDE FOR ITS STUDY}

\begin{abstract}
This article proposes a Reading guide for ESR through three dimensions (prevalent tool, use for management and ideological construction), which correspond logically and historically to ESR evolution. In effect, the state of knowledge and development of ESR in the last two decades is captured in a speech and in a series of current tools. The tool game for its application and assessment constitutes a useful dimension for management. Speech corresponds to ideological and doctrinal dimensions; thus, these are linked in management praxis. These dimensions allow comprehending ESR content and form. In other terms, this tridimensional theoretical division is a reading guide for ESR study. It is concluded that besides enterprise social responsibility progress, there is a contradiction between its humanist/ecologic vocation and private interest.
\end{abstract}

Keywords: Enterprise social responsibility, management useful dimension, ideological dimension, totality, organizational practice.

* Resultado de investigación del proyecto de postdoctorado en el Groupe international de recherche en éthique financiére et fiduciarie de la École des sciences de la Gestion de la Université du Québec a Montréal (Canadá).

** Ricardo Cuevas Moreno es un mexicano, Dr. en Ciencias de la Administración (IAE, Corse, Francia, 2003) Profesor investigador por la UAT-FCAT (MEX) y Candidato al Postdoctorado (ESG, GIREF) de la Université du Québec à Montreal financiado por el CONACYT (Mex, 2010). Está investigación se concluye durante el desarrollo de dicho postdoctorado.

Correo electrónico: rcuevasfr@yahoo.fr 


\title{
DIMENSÕES DA RESPONSABILIDADE SOCIAL DAS EMPRESAS (RSE): UMA GUIA DE LEITURA PARA ESTUDÁ-LA
}

\begin{abstract}
Resumo
Este artigo propõe uma guia de leitura para o estudo da RSE em três dimensões (ferramenta de atualidade, utilidade para a administração e construção ideológica) que têm evolucionado logicamente e historicamente com a RSE. Na atualidade, o estado de conhecimento e desenvolvimento da RSE nas últimas duas décadas se manifesta em um discurso e um conjunto de ferramentas. $\mathrm{O}$ conjunto de ferramentas para aplicá-la e avaliá-la é constitui a dimensão útil à administração. As outras duas dimensões (ideológica e doutrinária) correspondem ao discurso; portanto, se entrecruzam na prática da administração. Estas dimensões permitem compreender o conteúdo e a forma da RSE. Em outras palavras, esta divisão teórica tridimensional é ao mesmo tempo uma guia de leitura para o estudo da RSE. Concluímos que, apesar dos progressos na responsabilidade social corporativa, permanece uma contradição entre sua vocação humanista/ambientalista $e$ o interesse privado.
\end{abstract}

Palavras chave: Responsabilidade social da firma, dimensão útil da gestão, dimensão ideológica, totalidade, prática organizacional.

Cuevas, R. (2011). Las dimensiones de la responsabilidad social de las empresas: una guía de lectura para su estudio. En: Revista de la Facultad de Ciencias Económicas de la Universidad Militar Nueva Granada. rev.fac.cienc.econ, XIX(1).

JEL: M14, M18, M49.

\section{Introducción}

El presente trabajo es el resultado de un proceso de investigación sobre la ética de los negocios (EN) y la responsabilidad social de las empresas (RSE) iniciado hacia el año 2000 en Francia, continuado sucesivamente en México y en Canadá. La difusión y adaptación de la responsabilidad social por parte de las empresas y las organizaciones crea una profusión casi incalculable de estudios, artículos, libros, centros especializados, cursos y páginas Web. Este auge de la RSE para la empresa y las organizaciones contrasta con la falta de acuerdo en la definición de su objeto de estudio ${ }^{1}$. Vogel $(2005,2008)$ Lee, M-D (2008) y Ramboarisata (2009) a través de una extensa revisión de la literatura observan con justeza, esa falta de consenso entre los investigadores. La CEE define la RSE diciendo que ésta “...es la integración voluntaria de las cuestiones sociales y del cuidado del medio ambiente" (C.C.E., $2001,7)$, por parte de las empresas, la Norma ISO 26000 (2010) propone una definición de Responsabilité Social des Organizaciones (RSO) la cual busca ser la base de la línea directora para la comprensión e implementación de la RS en el mundo: “... responsabilidad de una organización frente a frente los impactos de sus decisiones y actividades sobre la sociedad y el medioambiente, traduciéndose en un comportamiento moral y transparente que contribuye al desarrollo sustentable, que incluye la salud et el bienestar de la sociedad; toma en cuenta las expectativas de los participantes de la empresa, respeta la leyes en vigor y que esté en acuerdo con las normas internacionales de comportamiento; et que es integrado en la organización en su conjunto y puesto en obra en sus relaciones" (ISO/DIS 26 000, 2009, 4,

1 Bien que existe la aceptación del triple bottom line (dimensiones económica, social y del cuidado del medio ambiente) ésta división aún no se enuncia como un objeto de estudio para la investigación de la RSE; esa clasificación se comprende más bien como una objetivo a alcanzar, es decir, cómo un equilibrio entre los objetivos económicos, sociales y del respeto al medio ambiente por parte de las empresas. 
8). Está definición contiene los aspectos moral, social, jurídico y ecológico sin alcanzar la totalidad de relaciones de la praxis de la empresa como veremos en las páginas siguientes.

Sin embargo, tal que lo subrayan Cuevas (2003; 2007; 2010) y Vogel (2005, 2008) la RSE algunas veces es adoptada, otras tantas, es impulsada por las presiones de las ONG's, los trabajadores, los clientes, o por las condiciones propias de cada industria en un contexto histórico, social y cultural donde surge el renovado interés por la ética. Nosotros pensamos que el objeto de estudio de la RSE debe provenir del movimiento mismo del fenómeno y no de una definición a priori impuesta como supuesto de investigación. En ese tenor una guía de lectura para su estudio se hace necesaria. Por tal razón, la secuencia lógica de este razonamiento establece las preguntas ¿Qué dimensiones forman la RSE? Si tales dimensiones existen ¿Cómo se constituyen y cuál es su función para la administración de la empresa en la actualidad?

Para dar respuesta a esas interrogantes debemos, tal como lo enseña Kosik (1970) "dar un rodeo". En efecto, la RSE representa una ruptura más en relación al paradigma neoclásico en economía. Taylor (1986; 1911) Fayol (1916) Simon \& March (1958, 1959) Alchian \& Demsetz (1972) Peters \& Waterman (1983; 1982) y North (1993; 1994; 1996) rompen cada uno a su modo, con la teoría económica estándar. Ellos representan algunas de las corrientes del pensamiento económico y administrativo las cuales en su conjunto apuntan a considerar la empresa capitalista y las organizaciones cómo una totalidad estructurada de relaciones económicas, políticas, sociales, culturales, éticas, ideológicas, institucionales y simbólicas; con sus objetivos a esos registros correspondientes (Cuevas \& Arroyo, 2006; Cuevas, 2011). La existencia misma de la RSE como cuerpo teórico y panoplia de útiles continúa con esa separación. En la empresa capitalista la búsqueda de la ganancia prima sobre los restantes objetivos sin significar que los produzca o los determine "en última instancia". Así la RSE significa un cambio de registro hacia la ética y en particular el valor moral de la responsabilidad. Sin embargo, el hecho que la ética y la responsabilidad se encuentren en el contexto de la producción de ganancia privada hace entrar a la RSE en una serie de contradicciones insuperables que nosotros llamamos verdadero problema de la RSE. Ahí la vocación humanista, social y del respeto al medio ambiente se encuentra en contradicción con el trabajo alienado o producción de beneficio. La RSE estudia -según nosotros- esa totalidad de relaciones entre los hombres quienes crean la empresa capitalista.

Esas ideas son los supuestos de nuestro presente trabajo. En concordancia con el método que profesamos, dichos supuestos se comprueban a través de la exposición. Esta investigación aborda la RSE movilizando distintos niveles de análisis y se desmarca de los discursos normativos, instrumentales y emergentes (Ramboarisata, 2009) por movilizar la dialéctica materialista en su estudio.

La estructura de la exposición se divide en tres partes. En la primera se establece el objeto convencional de la RSE y se transita hacia la parte segunda. Esta última expone los útiles con los que cuenta la RSE para su implementación dimensión de útil de la administración. La tercera parte expone la dimensión ideológica y doctrinaria de la RSE. Se concluye que la RSE juega un papel de importancia trascendente y creciente en el modo de vida capitalista actual y que esas dimensiones pueden servir como una guía de lectura para su estudio.

\section{Hacia un intento de definición de objeto de acción}

En la actualidad es un hecho comúnmente aceptado que las empresas deben ocuparse en forma creciente de su responsabilidad social frente a sus participantes (stakeholders); lo mismo acontece con la observancia de un comportamiento moral de parte de los administradores. Se considera que ambos aspectos se reflejan en la toma de decisiones eficaces y moralmente aceptables. Por ende, la empresa que actúa de manera socialmente responsable tiene una ventaja competitiva en la arena de la competencia (Porter \& Kramer, 2002). La consecuencia de este razonamiento es que la adopción de la RSE crea nuevo valor (Vogel, 2008, 34-37). 
En el modo de vida capitalista esta importancia por la ética y moral adquiere una connotación propia debido a la generalización de este modo de producción y su difusión mediante la economía de la informatización o si se prefiere globalización (Cuevas, 2007). El lenguaje común habla de una eclosión de "éticas": ética financiera, bio-ética, ética económica, ética de la empresa. Éstas son unas tantas formas de la ética aplicada para regular y provocar el comportamiento moral de los individuos y las empresas involucradas en esas actividades.

A partir de 1985 se difunden desde los Estados Unidos y las empresas transnacionales el Business Ethics. Paralelamente y durante la década de 1990 hasta la actualidad se propaga -bajo el impulso de la instituciones de Nacionaes Unidas, ONU, PNUD y PNUE- la doctrina del Desarrollo Sustentable (DS) cuya adaptación por las empresas deviene RSE. A la hora actual existe el consenso que se trata de una nueva disciplina formando parte de las ciencias de la Administración. Se trata de lo que en castellano se pude nombrar administración moral de la empresa y los negocios, responsabilidad corporativa o simplemente RSE. Esto porque siendo la Ética la ciencia de los juicios de valor a ella corresponde estudiar fenómenos como la Ética de los Negocios (EN) o la RSE. Mientras que "la administración moral" se refiere a la acción del pilotaje de la empresa haciendo caso de las leyes, las normas, los principios y los valores morales.

Sin embargo, existe la tendencia a hablar de RSE refiriéndose a la problemática de la moral en la administración de la empresa:
"La ética de los negocios se convirtió en negocios y sociedad o responsabilidad social de la empresas" (MacMahon, 2001, 418-420 en Frederick, 2001).

La certitud de esa aseveración es posible sustentarla de una manera sencilla. Obsérvese los temas tocados por la EN, el DS y la RSE: La RSE como reflejo o adopción del DS contendría los temas propuestos por la EN además de la dimensión social y de cuidado del medio ambiente ausente en el primero. Esta reducción no es del todo anárquica. Son las empresas las que hacen esta delimitación de lo que concierne a la RSE, pues en la práctica ellas hacen una adaptación e implementación de los temas propuestos.

Donalson \& Dunfee (1994, 252-254) clasifican en dos grandes vertientes la RSE. Por un lado, existen los partidarios del "acercamiento empírico". Éstos buscan solucionar problemas concernientes a la dirección la toma de decisiones, el marketing, los resultados financieros, la estructura de la organización y problemas de adaptación. Por el otro, están los partidarios del acercamiento normativo. Éstos establecen ideas sobre las prácticas y la dimensión moral de los negocios que no necesariamente existen aún. En consecuencia, Donalson \& Dunfee proponen un acercamiento integrador del business ethics (1994). Ciertamente, en el contenido de la RSE existen dos dimensiones:

Tabla 1. Temas abordados por EN, DS Y RSE ${ }^{2}$

\begin{tabular}{|c|c|c|}
\hline $\begin{array}{l}\text { ÉTICA DE LOS NEGOCIOS } \\
\text { (1985) }\end{array}$ & $\begin{array}{l}\text { DESARROLLO SUSTENTABLE } \\
(1972-2008)\end{array}$ & $\begin{array}{l}\text { RESPONSABILIDAD SOCIAL } \\
\text { DE LA EMPRESA (1970-2010) }\end{array}$ \\
\hline $\begin{array}{l}\text { Comportamiento Moral del } \\
\text { administrador } \\
\text { Recursos Humanos } \\
\text { Recursos } \\
\text { Relaciones con los stakeholders }\end{array}$ & $\begin{array}{l}\text { Cuidado del Medio Ambiente } \\
\text { Eficiencia Económica } \\
\text { Empleo } \\
\text { Justicia Social } \\
\text { Derechos del Hombre } \\
\text { Lucha contra la corrupción } \\
\text { Salud }\end{array}$ & $\begin{array}{l}\text { Eficiencia Económica } \\
\text { Empleo } \\
\text { Derechos del hombre } \\
\text { Condiciones de trabajo } \\
\text { Cuidado del Medio Ambiente } \\
\text { Relaciones con los stakeholders } \\
\text { Salud } \\
\text { Cultura }\end{array}$ \\
\hline
\end{tabular}

2 Elaboración propia. 
- la de útil para la administración

- la dimensión ideológica y doctrinaria

En las páginas siguientes se estudian estas dimensiones del contenido de la RSE con lo cual se pretende llenar una laguna y proponer una guía de lectura para su estudio.

\section{La RSE como útil de la administración}

Dentro del modo de vida capitalista todos los conocimientos, recursos físicos y humanos son puestos al servicio del capital o a la obtención de ganancia (Marx, 1867, 1965). Esta idea fundamental y justa por corresponder al modo de vida capitalista debe precisarse. En la actualidad, la empresa trata de cumplir además de sus objetivos económicos, los objetivos sociales y del cuidado al medio ambiente (triple bottom line). Y en efecto, para conseguir esas finalidades la empresa moviliza todos los conocimientos, el saber y la experiencia acumulada de las fuerzas productivas de la humanidad, incluyendo en éstas a la naturaleza. Por tal razón, desde su inicio la EN y la RSE se convierten en un útil de la administración; la ética aplicada a la empresa es utilizada como un objeto para la creación de valor como cualquier otro conocimiento.

Ciertamente, si la RSE atrae de principio a los administradores y capitalistas es porque ella puede dar mayores beneficios, evitar pérdidas, embellecer la imagen y asegurar la perennidad de la empresa ${ }^{3}$. En su implementación, la RSE crean una serie de útiles que en conjunto pretenden incrementar el performence de la empresa. Estos instrumentos al igual que la EN, DS y la RSE mismas son una construcción social la cual constituye un todo único.

El juego de útiles está constituido por las Cartas y Códigos Éticos, los Informes Sociales, las líneas de dirección planteadas por ejemplo por el Global Reporting Initiative (1997), OCDE (2008) ISO 26000 (2010) Chambre de Commerce internationale (1991), los índices de DS como el ASPI, Ethibel Excellence y Ethibel Pioneer y Dow Jones Sustainability Indexes, las Normas como las ISO 14000, AA 1000 (2003), SA 8000, y las Auditorias Éticas ${ }^{4}$. Estos útiles diseñados por organismos internacionales y oficinas especializadas se integran a la administración estratégica y operacional de la empresa. En conjunto los especialistas los nombran "papeles éticos".

\subsection{Cartas y Códigos Éticos}

Estos documentos contienen la enunciación de la misión, la visión, los valores y principios que la empresa considera los más importantes para su gestión y sus relaciones con sus participantes (stakeholders). Éstos surgen con "la ola ética" de mediados de los ochentas y al parecer son los más difundidos en las empresas y las organizaciones pues, éstas sean grandes o pequeñas los enuncian. Vogel $(2006,2008)$ en su obra compendiosa ubica la difusión de los "códigos voluntarios" durante la década de los noventas en las industrias del calzado, artículos deportivos, los juguetes, el vestido, la agricultura (producción de café) en los países en desarrollo para regular las condiciones de trabajo y evitar el trabajo de los niños.

La primera de las Cartas éticas es la de empresa Penny Company (1913). La segunda es la de Johnson \& Johnson (1943) ambas pueden considerarse como los antecedentes de los documentos éticos. Las cartas éticas contienen los principios, los valores y la identidad de la empresa (Gélinier, 1986) Este pionero de la ética de los negocios en Francia, autor piensa que en los códigos de las empresas americanas se recuerdan las leyes y dan las reglas concretas para la administración con la

3 Vogel $(2006,2008)$ establece con elocuencia los límites de esas ideas; es decir sus alcances. En su amplia revisión de literatura especializada, el autor establece que existen pocas pruebas empíricas que sostengan esas ideas pero -según él- las empresas y los administradores actúan como si fueran ciertas.

4 Algunas direcciones donde pueden consultarse esos útiles son: www.globalreporting.org, www.iccwbo.org/index_sdcharter.asp, www.sustainability-index.com, www.accountability.org.uk

5 Tal vez se llama "papeles éticos" para destacar la dimensión moral de estos documentos pero en realidad no pertenecen a la Ciencia de la Ética aunque pueden ser objeto de estudio de ésta. 
finalidad de resolver las interrogaciones morales, remitiendo a principios de carácter general. Mientras que las empresas francesas tratan de integrar los valores morales en sus cartas y códigos éticos. Esto es una diferencia de forma pues la función para la cual los códigos y cartas éticas son creados en ambos continentes es la misma. En primer lugar, estos documentos pretenden ser una guía de acción asegurando el comportamiento moral del administrador y los empleados. Esto para dar un mejor servicio o producto asegurando el éxito frente a la competencia y así obtener mayores beneficios. En segundo lugar, los códigos éticos pretenden regular el comportamiento de la empresa frente a sus participantes (stakeholders). La forma en cómo esas ideas se presentan depende de la empresa misma (Naudet, 1993).

Los códigos éticos son diferentes a los códigos deontológicos. Como su nombre lo indica, estos últimos son las reglas de conducta que una profesión, por ejemplo los contadores públicos, los establecen para regular el comportamiento moral de sus colegas.

\subsection{Informes de Responsabilidad Social de la Empresa o Desarrollo Sustentable}

Según D'Humières (2005) el Informe de Responsabilidad Social (IRSE) o Informe de Desarrollo Sustentable es el documento por excelencia de la comunicación de las empresas internacionales y las organizaciones. Esto por varias razones. En primer lugar, al englobar las dimensiones económicas, social (derechos del hombre) y del cuidado al medioambiente, el informe de RSE busca presentar al conjunto de los participantes de la empresa (stakeholders) y al público en general, un panorama completo de la situación, la implementación y resultados del DS en las organizaciones. En segundo lugar, el IRSE al presentar los objetivos, avances y compromisos de la empresa frente a sur participantes, por lo cual el IRSE está directamente ligado su estrategia. En tercer lugar, muestra la implementación del DS como proceso posible de ser comprendido a través del tiempo. En cuarto lugar, una comprensión más profunda del papel de este informe reside en que es el lugar donde se rinde cuentas, se valida y explica como el círculo virtuoso del sistema que parte del triple bottom line crea valor para los participantes de la empresa; es decir, explica cómo una preocupación moral crea valor.

El Global Reporting Iniciativa (1997) es el organismo internacional que impulsa el DS poniendo a disposición a nivel mundial el conjunto de conceptos, los indicadores y forma de presentar el Informe de Desarrollo Sustentable de las empresas. Su misión es proporcionar las organizaciones de cualquier lugar, tamaño y sector un marco confiable y creíble para dicho informe (GRI, 2006, 2). Este organismo es resultado de la Coalición de las Economías del medio ambiente responsables (CERES) en asociación con el Programa de Naciones Unidas para el Medio Ambiente (PNUD).

Con la contribución de empresas trasnacionales y asesores en las áreas económicas, sociales y del medio ambiente, esta coalición propone la Guía para la elaboración de Memorias de Sostenibilidad (2000-2006). Este trabajo es bastante extenso pues, sugiere de manera detallada los conceptos, objetivos y parámetros para medir, demostrar y comparar el desempeño de la organización en materia de DS. Esta rendición de cuentas de la empresa frente a la sociedad tiene entre sus características importantes la transparencia, pues pretende comunicar de manera "clara y abierta" los resultados de las empresas que adoptan la vía de durabilidad. Otros principios que sostienen la Guía son la pertinencia, la exhaustividad, el equilibrio, la comparabilidad, la claridad y la fiabilidad. Éstos principios que la misma Guía define en su primera parte junto con los parámetros e indicadores por ésta propuestos y la manera de presentar el Informe de DS, le brindan a ésta consistencia y rigor. Estas características en su conjunto posicionan al Global Reporting Iniciativa (1997) muy próximo a su misión de ser el marco de referencia a nivel mundial para toda empresa de cualquier tamaño y sector.

El Informe de Responsabilidad Social es válido no solo porque rinde cuentas de manera transparente y de acuerdo a los principios arriba señalados sino porque se encuentra en concordancia con las normas vigentes. En la literatura del DS y la RSE se hace continua referencia a las normas, que al igual 
que la Guía para la elaboración de Memorias de Sustenibilidad (2000-2006) pretenden un alcance mundial.

\subsection{Normas para el DS}

International Organization for Standardization (ISO, 1947) es el organismo cuya finalidad es establecer normas internacionales para la industria y el comercio. Continuando con esta tradición la ISO establece la serie de Normas para al menos 200 rubros entre los cuales las normas ISO 14001 dictan los criterios para la reducción de los efectos negativos de la actividad de las empresas sobre el medio ambiente.

Al igual que en las otras series de normas ISO las empresas grandes o pequeñas, las organizaciones de todo tipo pueden conseguir la certificación de la norma mediante otro organismo certificado por la propia Organización Internacional de Normalización. Un ejemplo práctico puede dar cuenta de cómo la Norma ISO 14001 es obtenida. Metivier (2000, 306-307) explica el proceso de certificación del Sistema de Administración del Medio Ambiente (SAM) de una empresa del sector farmacéutico: Previo una auditoria para conocer la situación un plan de acción elaborado por asesores, es ejecutado.

Pese a al prestigio de Organización Internacional de Normalización (ISO, 1947) y su pertinencia para asegurar la calidad de la administración del medio ambiente dos críticas sobre la serie de normas ISO 14000 merecen ser señaladas. Primero ellas no establecen de manera explícita la búsqueda de un DS para la empresa y las organizaciones. Segundo, el hecho que las organizaciones consigan la certificación no significa que dejen de contaminar.

Tabla 2. Normas ISO sobre la RSE ${ }^{6}$

\begin{tabular}{|c|c|}
\hline NORMA & ADMINISTRACIÓN DEL MEDIO AMBIENTE \\
\hline ISO 1401 & Sistema de Administración del medio ambiente- Especificaciones y líneas directrices para su uso \\
\hline ISO 1404 & $\begin{array}{l}\text { Sistema de Administración del medio ambiente- Líneas directrices generales referentes a los prin- } \\
\text { cipios, sistemas y técnicas para su ejecución }\end{array}$ \\
\hline ISO 14011 & Evaluación del medio ambiente y sitios de los organismos \\
\hline ISO 14031 & Administración del medio ambiente- Evaluación del desempeño- Líneas directrices a seguir \\
\hline ISO 14015 & $\begin{array}{l}\text { Administración del medio ambiente- Ejemplo de la evaluación del desempeño sobre el medio am- } \\
\text { biente }\end{array}$ \\
\hline ISO 14050 & Administración del medio ambiente- Vocabulario \\
\hline ISO 14061 & $\begin{array}{l}\text { Información para ayudar a los organismos forasteros en la utilización de normas ISO } 14001 \text { y ISO } \\
14004\end{array}$ \\
\hline ISO/TR 14062 & $\begin{array}{l}\text { Administración del medio ambiente- Integración de los aspectos del medio ambiente en la concep- } \\
\text { ción y desarrollo del producto }\end{array}$ \\
\hline ISO 19011 & $\begin{array}{l}\text { Líneas directrices para la auditoria de los sistemas de administración de calidad y/o del medio } \\
\text { ambiente }\end{array}$ \\
\hline
\end{tabular}

6 Fuente: Wikipedia, L'encyclopédie libre, http://fr.wikipedia.org/wiki/S\%C3\%A9rie_des_normes_ISO_14000oui 
Tabla $\mathbf{3}^{7}$. Posibles etapas del proceso de certificación de la norma iso 14001

\footnotetext{
- Se identifican los impactos significativos (negativos y positivos) de la actividad y se disciernen los ejes de la política del medio ambiente en el sitio de producción.

- La documentación y la redacción del SAM son elaboradas a partir de la norma ISO 9002 ya existente en la empresa.

- Los auditores internos son capacitados mediante un programa ad-hoc.

- Los procesos y las actividades son escritos.

- Un programa de sensibilización para el personal es llevado a efecto a fin que en lo cotidiano se comprenda la necesidad de reducir los efectos nocivos sobre el medio ambiente.

- Se establece un nuevo programa de acciones de cuidado al medio ambiente, estableciendo las operaciones y los costos.

- Revisión del SAM por las auditorías internas. Luego su análisis operacional con una auditoria "en blanco".

- En base a esos resultados un asesor ayuda a la movilización del personal para la obtención de la certificación.

El tiempo estimado total de todo este proceso es de un año.
}

Ante tales defectos y ante la necesidad de definir la RS de las organizaciones, sus principios e líneas de implementación ISO (2010) lanza la Norma 2600. Este trabajo atípico desde su concepción y elaboración par la intervención de multi-participantes, 90 países y 40 organizaciones pretende contribuir a la concepción et implementación de la RSO estableciendo una panoplia de conceptos y juicios de alcance mundial. Sin pretender ser una norma para la certificación el efecto de ISO 26000 depende de su adopción por parte de las organizaciones.

\subsection{Auditorías sobre el DS de las organizaciones}

La Auditoría sobre el DS o la RSE es un proceso sistemático, objetivo e independiente cuya finalidad es determinar cómo la actividad de la empresa o la organización satisfacen las exigencias de su dominio. El auditor puede ser un individuo o una organización. En Francia por ejemplo, Vigeo es un organismo especializado en la realización de Audits los cuales son definidos como sigue: Una auditoria en Responsabilidad Social permite identificar la naturaleza de los riesgos y su nivel de dominio por parte de la organización auditada. El grupo Vigeo mide el nivel de compromiso de las organizaciones en relación a los objetivos de Responsabilidad Social que constituyen, por ellos mismos como para sus participantes (stakeholders) facto- res de riesgo (www.vigeo.com/csr-rating-agency). La auditoria de responsabilidad social es además, un útil operacional que ayuda en la toma de decisiones. Esto para mejorar los proceso, optimizar los trabajos de los equipos y la planificación estratégica, crear nuevas áreas de oportunidad y ajustarse a los principios de la Agenda 21. En fin, la auditoria del DS o la RSE es una herramienta de control.

Viego ofrece una serie de servicios que van desde las auditorias sobre la política del cuidado al medio ambiente, pasando por los medios y los resultados de esa política hasta y una auditoria en concordancia con la Diez Principios del Global Compaq de la ONU. Las principales ventajas de un proceso de auditoría de RSE hecha por un organismo como Vigeo son:

1. La independencia y la objetividad del proceso de auditoría.

2. El aseguramiento de su calidad gracias a la asesoría de un comité de expertos en las tres dimensiones de la RSE.

3. El aseguramiento de su calidad gracias a una metodología y parámetros para medir el desempeño de la organización en materia de RSE.

4. El proceso ofrecido por Vigeo, sigue las recomendaciones, de las Convenciones y Principios Directores de la ONU, OCDE y la OTI.

7 Fuente: Elaboración propia a partir de Metivier (2000). 
En general la implementación de la RSE significa un gasto para la empresa. Para las empresas un tipo de asesoría y auditoria como la del ejemplo de este apartado significa un costo el cual puede ser cubierto por las grandes empresas mientras que ese costo puede ser un impedimento para las pequeñas empresas.

\section{5. Índices sobre el Desarrollo Sustentable e Inversión Socialmente Responsable}

Los Índices sobre el Desarrollo Sustentable tienen por objetivo ponderar el desempeño de la política de RSE sobre el rendimiento financiero a partir de una fecha determinada. Esto es de suma importancia desde el punto de vista de la generación del valor, pues la implementación de DS se justifica a condición que dicha vía demuestre un aumento del valor financiero. Esclarecer la veracidad de esa correlación es fundamental para la RSE y la EN. Bien que no se ha comprado científicamente, se cree que los Índices sobre el Desarrollo Sustentable o Índices de RSE ilustran los cambios en el valor financiero de las empresas socialmente responsables. Sin embargo, en un segundo momento, dichos índices tienen por vocación poner en relieve las empresas cuyas prácticas de responsabilidad social son más elevadas según las agencias especializadas, más que la correlación económica-financiera de DS y valor financiero (D'Humières, 2005, 363).

El primer índice sobre DS y valor financiero es creado por KLD Research \& Analytics, Inc ${ }^{8}$, agencia pionera en la investigación y herramientas de la Inversión Social Responsable (ISR) en EUA (D'Humières,2005; Laville, 2006; Vogel, 2008). En el Domini 400 Social Index (1989) cotiza a las empresas que respetan criterios del cuidado al medio ambiente, sociales y de gobierno de la empresa (siglas en inglés ESG); genera además una familia de índices del DS. Su objetivo es ser un referente para los inversionistas. Luego, Dow Jones en asociación con el administrador de fondos de IRS Sustainability Asset Management (SAM) crean otra familia de índices llamada Dow Jones Sustainability Indexes (DJSI) $^{9}$ (1999) los cuales son considerados como los más importantes del género. Éstos tienen un alcance mundial como DJSI- World, regional como el DJSI Stoxx and Euro-Stoxx y por país como el Dow Jones Sustainability North America Index (DJSI North America) y el Dow Jones Sustainability United States Index (DJSI United States). Después y en las mismas dimensiones (país, regional y mundial) la agencia Ethical Investment Research Service (EIRIS) en asociación con Financial Times Stock Exchange (FTSE) (2001) grupo especializado en el cálculo y difusión de índices bursátiles crean la serie de índices FTSE4Good ${ }^{10}$. Paralelamente, la agencia (ARÈSE) lanza el índice ASPI Eurozone ${ }^{\circledR}$, administado por Vigeo ${ }^{11}$. Esta última empresa ofrece además los índices Ethibel Excellence y Ethibel Pioneer para ser integrados con facilidad en el Informe de Responsabilidad Social.

En relación a la metodología de construcción, los Índices sobre el Desarrollo Sustentable son -por su naturaleza- índices sintéticos pues deben contener los distintos parámetros del desempeño (performence) de la empresa en las dimensiones social, del cuidado del medio ambiente y de gobierno de la empresa.

En primer lugar, Índices sobre el Desarrollo Sustentable e Inversión Socialmente Responsable son construidos a partir de un índice "clásico" de referencia; por ejemplo Domini 400 Social Index (1989) del índice bursátil S\&P 500 de la filial de análisis financieros de McGraw-Hill llamada Standard \& Poor's. Otro ejemplo, es el Dow Jones Sustainability Indexes (DJSI) del Dow Jones Global para el mundo (DJGI World) (Laville, 2006, 116) el cual cotiza 600 empresas.

\footnotetext{
http://www.kld.com/

http://www.sustainability-index.com/

http://www.ftse.com/Indices/FTSE4Good_Index_Series/index.jsp

$11 \mathrm{http}: / /$ www.vigeo.com/csr-rating-agency/fr/offre-de-services-rse/vigeo-group-rating/communiquez-sur-votre-presence-dans-unindice-de-developpement-durable.html
} 
El segundo aspecto en su construcción es el universo de referencia (número de empresas contenidas). Por ejemplo, Domini 400 Social Index trata de contener 250 empresas a partir del universo de $S \& P$ 500. En ese universo se encuentran excluidas las empresas dedicadas al alcohol, tabaco, armas de fuego, juegos de azar, la energía nuclear y las armas militares. De igual forma quedan fuera, las empresa que no cumplan con los estándares financieros propuestos por KLD (capitalización, ganancias, liquidez, precio de acciones, deuda). Otro ejemplo, son los índices ASPI Eurozone ${ }^{\circledR}$, Ethibel Excellence y Ethibel Pioneer. El primero tiene 120 empresas cotizadas en la zona Euro, los otros dos contienen alrededor de 280 empresas cada uno de Europa, EUA y Asia- y Pacífico.

Otro aspecto a considerar sobre el universo, es la zona geográfica. La empresa puede estar contemplada en una región particular como la Zona Euro y pertenecer al mismo tiempo a otra zona o no serlo. Así mismo, una misma empresa puede aparecer en varios índices. Pero en la actualidad no aparecer en alguno de esos índices puede significar un mal síntoma para los inversionistas y los consumidores. Las dimensiones sociales, del cuidado al medio ambiente y el gobierno de la empresa son desagregados en distintos parámetros según la metodología particular de la agencia creadora del índice.

Los Índices sobre el Desarrollo Sustentable (Índices Éticos o Índices de RSE) son un útil de un sistema más amplio. La Inversión Socialmente Responsable (ISR) o Inversión Ética o Inversión Desarrollo Sustentable, surge al momento de la renovación de la RSE durante el decenio de los años 1990's. Bien que los Fondos éticos o Fondos de Inversión Socialmente Responsable (FISR) datan desde la década de 1920 en EUA con los fondos religiosos, la ISR y los FISR tienen la particularidad de tomar en cuenta además de los criterios económicos y financiero, los sociales (justicia social, respeto de los derechos del hombre, respeto de los derechos laborales), del cuidado al medio ambiente y de gobierno de la empresa.

La cuestión de los Índices sobre el Desarrollo Sustentable e Inversión Socialmente Responsable que pretenden dar cuenta de la creación de valor financiero por la RSE pertenece a una problemática general que es la relación entre el Performance Social de la Empresa (PSE) y el Performance Financiero de la Empresa (PFE). Una larga discusión después de los trabajos pioneros de Moskowitz (1972), Sethi (1975); Carroll (1975; 1979), O'Bannon \& Preston (1993); Frederick (1986); Aupperle et al. (1985), Wartick \& Cochran (1985), Jones (1980), Ullmann (1985); Clarkson (1995); Wood (1980); Epstein (1987) McWilliams \& Siegel (2000) pasando por met-análisis como los de Griffin \& Mahon (1997), Margolis \& Walsh (2003), Orlitzky et. al (2003) e intentos de síntesis como los de Maron (2006), Crifo \& Ponssard, (2008) o Callan \& Thomas (2009) muestran la imposibilidad de una conclusión definitiva a saber si la RSE crea valor económico o financiero para la empresa ${ }^{12}$.

En suma, los útiles de la RSE, aunque surgidos de distinta fuente $y$ en diferentes momentos son puestos en práctica por las empresas constituyendo una verdadera panoplia que viene a unirse a las herramientas tradicionales de la administración. Sin embargo, existen otras dimensiones que pasan hasta cierto punto desapercibidas por los investigadores pero que forman parte de la RSE.

\section{La dimensión ideológica y doctrinaria de la administración moral}

Durante algunos años, Cuevas (2003, 2004, 2005, 2006, 2007, 2008, 2011) estudia la dimensión ideológica contenida la RSE. Su tesis doctoral dedicada a ese tema, explica cómo se construye la ética de la empresa y cómo gracias a esa construcción ésta puede funcionar como ideología en el sentido de Marx \&

12 En lengua inglesa una pléyade de trabajos de la relación entre PSE y PFE es objeto de nuestra investigación posdoctoral. Margolis \& Walsh (2003) cuentan 127 artículos sobre ese tema entre 1972-2002. Una revisión en la base de datos EBSCO de la UQAM cuenta alrededor de 95 artículos entre 2003-2011. 
Engels (1982). Usando una metáfora, se pude decir que la RSE es como un ensamble de conocimientos y el saber de distinta procedencia al servicio de la reproducción del capital en todos los órdenes. En esas aportaciones se encuentran contenidos, el conocimiento, el saber y la experiencia de los asesores e investigadores (economistas, administradores, filósofos, psicólogos, sociólogos, abogados) dirigentes de empresas y organismos internacionales.

La evolución del tema ético para empresa con el auge del DS y la RSE conduce a establecer esas fuentes con la importante aportación de los organismos internacionales. Al propio tiempo, se precisa la comprensión de las dimensiones ideológicas y doctrinarias de la Ética de la Empresa (EN) y la Responsabilidad Social de la Empresa (RSE).

En efecto, Faber (1992, 191, 192, 243) desarrolla la idea que la EN contiene una dimensión ideológica al grado de constituir "un golpe bajo a la sociedad". Y es que la empresa confisca la moral para permanecer eterna mientras que ésta (la empresa) debe estar sujeta a la ley de la ganancia. Según Faber los administradores son los principales beneficiarios de "esta corrupción en el sentido de las palabras": Esto es cierto. Sin embargo, el libro de Faber tiene deficiencias mayores que le impiden derivar las consecuencias que el camino sobre la ideología conlleva. La primera es que a pesar de la justeza sus aseveraciones, él confunde la categoría de ideología con la noción de sofisma. Y es que la ideología del capital para la empresa es algo más complejo que un engaño con la sutileza del lenguaje. La segunda deficiencia es que los principales beneficiarios no son solamente los dirigentes de la empresa sino la clase capitalista y las capas de clase que a ésta se adhieren. A nivel mundial, los beneficiarios de esa tergiversación del lenguaje que refleja la apropiación real de la moral para la producción de ganancia, es la oligarquía burguesa mundial. En el origen de esas deficiencias del libro de Faber se encuentra la ausencia de la categoría de capital como relación social.

Este trabajo de Faber y otros como el Marcuse (1964), Hayek, Leroux (1995), Landier (1991), Floris (1996) Denzau \& North (1994) son un aleja- miento de la categoría de ideología pero al propio tiempo, la crítica de estos trabajos marca el inicio del camino para rescatar esta categoría, tal como Marx \& Engels $(1982,1846)$ la formulan. Estos últimos retoman esa categoría del Comte de Tracy (1817, 1970) quien trata de estudiarla por primera vez de manera sistemática. Él la comprendía como la ciencia de las ideas vecina de la lógica, la gramática y especialmente de la psicología o la subjetividad. Para Marx \& Engels (1846, 1982) la noción de ideología adquiere una base material a partir de la propiedad privada y la división de la sociedad en clases sociales. En efecto, la ideología es la forma que adquieren las relaciones de propiedad, poder y dominio de una clase sobre otra (s). La ideología es la conciencia mixtificada de esas relaciones; ésta se cristaliza en el Derecho, la Política, la Filosofía, la Ética, la Religión y en toda forma de pensamiento que de manera voluntaria o involuntaria oculte esas relaciones de dominio pero al mismo tiempo las perpetúe. Para ser aceptada, ella toma la apariencia de verdad y aparece no como la ideología de una clase particular sino como la ideología común a todos los miembros del organismo social. Por eso en una época determinada, las ideas dominantes son las de la clase social materialmente y espiritualmente dominante (Marx \& Engels, 1846, 1982, 1080-1081).

Planteada así la categoría de ideología se convierte en un poderoso útil para comprender la realidad social. Empero, la ideología formulada así se encuentra en un nivel de generalidad que impide comprender cómo un fenómeno i.e. la RSE pueden funcionar como ideología para la empresa y la sociedad. Ello implica una análisis multinivel de relaciones económicas, políticas, sociales y culturales (Cuevas, 2007 , 2011). Al tomar esta vía se comprende no solo la dimensión ideológica y doctrinaria de la RSE sino su origen, constitución y funcionamiento, es decir, su construcción como objeto de investigación científico. En este sentido es necesario precisar algunas ideas centrales, sobre la problemática de la RSE, la función de los valores morales en la administración de las empresas y la difusión de la ideología del capital para la empresa. En trabajos anteriores Cuevas (2003, 2005, 2006, 2007, 2008, 2009) responde a esas cuestiones. Aquí baste recordar lo siguiente: 
A. En efecto, la problemática de la RSE es algo más que una conjugación armónica del triple bottom line. Por un lado, la vocación humanista y comunitaria de la RSE se ve confrontada con la apropiación individual y los beneficios de los productos del trabajo. En otras palabras, la búsqueda del bienestar social se encuentra en contradicción con el trabajo alienado que es el fundamento de la sociedad burguesa. Por el otro lado, al tomar decisiones en el contexto de ese usufructo privado-individual, las empresas capitalistas determinan más que nunca el destino del hombre moderno. Ésta dualidad, es la verdadera problemática de la administración moral de la empresa y los negocios o RSE.

B. Entre las transformaciones que son la base real sobre la cual la RSE surge, la economía de la informatización es central. Justamente, los especialistas establecen que el cambio en las estructuras de las empresas hace de éstas disposiciones planas liberando espacios de autonomía y libertad para el individuo y los grupos de trabajo. Esos espacios son indispensables para el cumplimiento de los objetivos de los procesos de trabajo. En la literatura de la Administración el libro de El Precio de la excelencia (Peters y Waterman, 1982, 1983) marca el hito de ruptura con la administración tradicional. En esa nueva manera de gestionar la empresa en red los valores morales adquieren un papel primordial para alcanzar la excelencia. Así un sólido código moral es una condición indispensable para el éxito de la empresa.

C. Ex poss para poder funcionar como ideología la RSE debe por un lado, ser una expresión inversa o idealizada de las relaciones sociales de producción, poder y dominio; con apariencia de verdad gracias a ser enmascarada de ciencia o de filosofía (Marx \& Engels, 1846, 1982). En el mismo sentido, la ideolgia es una forma de pensamiento teórico que se desarrolla abstractamente sobre sus principios, presentándose como autónoma pero que es producto de las condiciones sociales y económicas (Lalande, 1926; 2002, 459 ). Por el otro lado, la RSE es un ensamble de conocimientos, de la Econo- mía, la Administración y la Ética; junto con el saber y la experiencia de los especialistas en el tema. Ambas condiciones son indispensables y constituyen la dimensión ideológica de la RSE.

D. El paso de lo general a lo particular es una operación lógica que debe corresponder al movimiento real de los fenómenos. Hablar de ideología en general-aún sea desde el punto de vista de su acepción vulgar como mundo de ideas- resulta inoperante para comprender cómo funciona la ideología que la RSE porta para la empresa. Para ese propósito es necesario comprender que "la empresa capitalista" crea un mundo de ideas, de creencias, de valores, principios y una imagen; los cuales aparecen como comunes para todos sus participantes. Esa es la ideología particular de la empresa.

E. La categoría de ideología formulada por Marx \& Engels $(1846,1982)$ explica la relación entre el poder económico y el primado ideológico de una clase sobre otras así como la forma que ésta relación asume en el conjunto del organismo social. Por tanto, esa explicación es válida. Sin embargo, las investigaciones recientes sobre la comunicación corporativa y el poder dentro de la empresa permiten comprender cómo ese proceso de creación, desarrollo, difusión y adopción de la ideología en particular tiene lugar.

Mintzberg (1986) explica en detalle las fases de creación de la ideología de la empresa. De igual forma, este autor observa la existencia de dos sistemas que permiten el establecimiento y difusión de la ideología. Según él, dentro de la organización los hombres deben realizar esfuerzos bajo una bandera común. Un factor importante para que esto tenga lugar lo es la ideología. No obstante, por su condición intangible la ideología no es tomada en cuenta por los administradores acostumbrados a la valoración de los hechos facticos y medibles. Para Mintzberg (1986, 221-222) la ideología se define como un sistema de valores y creencias al cual los miembros de la organización se adhieren. A pesar que Mintzberg evita establecer de manera categórica 
el nexo entre el poder nacido de la propiedad y la ideología que de este se deriva, en su explicación del funcionamiento de los sistemas ideológico y autoridad es posible apreciar esa relación.

En efecto, Según Mintzberg (1986) los propietarios de la empresa ceden al Consejo de Administración (C.I.) las facultades legales, económicas y financieras para la gestión de la empresa.

Luego, el C.I. hace lo mismo con el Presidente Director General (P.D.G.) quien a su vez delega poder y autoridad a los Directores (D). Éstos hacen lo mismo con los Administradores (A) empleados (E) y trabajadores (T). Se trata de una cadena de autoridad. Esta estructura es la base del sistema de autoridad y del sistema burocrático de comando y control. Mintzberg comprende de manera certera la necesidad de la ideología y su funcionamiento a través de lo que él nombra el sistema idelógico. Pues, la movilización, el control de los hombres en ese esfuerzo en común dentro de la empresa es imposible sin ese elemento inmaterial al cual los hombres se adhieren y que es la ideología.

Ésta última pasa por dos fases una de establecimiento y otra de refuerzo. El análisis de Mintzberg (1986, 234) es minucioso al punto de establecer los mecanismos de asimilación la ideología: (1) Natural y espontánea. (2) Selectiva por reclutamiento y promoción. (3) Adoctrinamiento y socialización, y (4) Voluntaria.

A partir de lo anterior, se entiende por que a cada puesto corresponde una cuota de jeraquía y una cuota de autoridad pero también una ideología que impulsa a los hombres a actuar y obedecer. De esta forma el análisis de Mintzberg prolonga y enrriquece el de Marx \& Engels (1846, 1982) sobre la ideología. No obstante, a pesar de su veracidad el estudio de Mintzberg -al igual que Faber y Floris- no llega a comprender que son los propietarios como capitalistas los detentores del poder, es decir, que la fuente del poder, la autoriad, la disciplina y el control es el capital como relación social.

Bien que este diálogo entre Marx, Engels y Mintzberg explica el por qué y cómo de la existencia de la ideología, no responde sino parcialmente a la cuestión de cuál es el contenido de la ideología de la empresa o qué es lo que se asimila y difunde.

En este sentido, el diálogo entre Marx y la comunicación corporativa da una respuesta. En efecto, la ideología de la empresa está constituida en una primera instancia, por los conceptos, valores morales, los juicios y razonamientos propios del modo de vida capitalista. Weber (1904-1905) muestra de manera magistral cómo las ideas del modo de vida burgués son verdaderas fuerzas históricas capaces por sí mismas -según él- de originar el capitalismo. Weber identifica esas ideas con los valores de moral ascética de la ética prostestante: empeño en el trabajo, abstinencia, tolerancia, acción racional y metódica.

Sin embargo, en la actualidad la ideología del capital es mucho más rica que en la época de Marx y Weber. Para los especialistas como Floris (1996) es apartir de los años ochentas que un viraje en la comunicación corporativa tiene lugar. Explicar las razones por la cuales esta comunicación de la empresa hace irrupción en el espacio público justo en ese momento es la problemática del libro de Floris (1996). Este trabajo representa una veradera genealogía de la emergencia de la ideología del capital para la empresa y la comunicación corporativa, para el dominio y la legitimación de la clase empresarial en Francia. Floris, con rigor lógico y apoyado en el desarrollo histórico de su sociedad muestra cómo la ideología del capital se destila e invade el espacio público.

Según Floris, en Francia, la comunicación corporativa y la hegemonía que ésta sustenta viene a ocupar el "vacio" dejado por la desaparición de la ideología de la lucha de clases, por un lado; y por el otro, la perdida de hegemonía de la organización síndical y el Estado, junto con el descredito de la empresa. Para el primado de la ideología de la cultura de la empresa sustituye luego entonces, a la idelología de oposción entre asalariados y patrones. En su lugar se instaura la competencia de empresas o grupos de empresas contra otras. Solo bajo esa base la empresa, su ideología, su proyecto y su cultura pueden englobar a todos sus participantes quienes desde 
ese momento deben asumir dicho proyecto somo el suyo propio.

En otro sentido, la transformación de la estructura de la empresa gracias a la informatica y las comunicaciones requiere una movilización de los equipos de trabajo con autonomía y libertad para la gestión de la información y la toma de decisiones (Floris, 1996, 88-89, 102-103, 106). Para Floris la comunicación corporativa tiene dos funciones mayores: (1) legitimar a la empresa en el espacio público. (2) Establecer una nueva realción entre los administradores y salariados. Ciertamente, el control y el concenso no solo requieren la movilización de las condiciones objetivas sino la aceptación subjetiva de los hombres. Por eso Floris habla de una administración de la subjetividad ${ }^{13}$ en la cuál la ideología del capital y la comunicación ocupan un lugar fundamental para el nuevo modelo de administración que este autor identifica con la administración participativa. Según Landier (1991, 56, 102) el surgimiento de ese modelo de gestión se explica parcialmente por la pérdida del poder de los sindicatos y la crisis de las formas tradicionales de su lucha.

Floris, influenciado por Bordieu y Godelier habla de una "legitimación simbólica" y de un "poder simbólico" refiriéndose a la legitimación y el poder cuya ejercicio reside en la subjetividad de los hombres que lo ejercen y los hombres que son objeto de éstos. Por eso, lo que el llama la idelogía del management (la ideología de la administración) en su ejercicio gracias a la comunicación, permite la reproducción de la relación de legitimidad, de dominiación y reproducción de la inigualdad en el espacio social. "La ideología de la administración" tiene las caracteristicas siguientes:

- A diferencia de las ideologías de los siglos pasados, la ideología de la administración está constituida por aportes de distintas fuentes. En efecto, Galbraith en (1967, IV) establece el rol de la teoría clásica del mercado en tanto que estratagema ideológico destinado a enmáscarar comadamente el ejercicio del poder en la gran empresa. Pero en la actualidad esto es solo una parte de los aportes para constituir la ideología del capital para la empresa: $L a$ dimension moral de Smith para la empresa $y$ los negocios, las teorías éticas, las nuevas teorías de la organización (teoría de los costos de transacción, la administración participativa, teoría de los participantes de la empresa, teoría de los recursos, la teoría del aprendizaje), las teorías éticas de los grandes filósofos, las Iniciativas, Conferencias y Declaraciones de la ONUPNUE, PNUD, OMC, OTI para la empresa, son las fuentes que constituyen la E.E., DS y la RSE enrriqueciendo al mismo tiempo, la ideología del capital para la empresa en la epoca actual (Cuevas, 2005, 2006, 2007, 2011).

- Existe una relación solidaria entre ideológia del capital y tecnología de la informática y las comunicaciones. En efecto, Cuevas (2007) establece la idea que esta última es la base real sobre la que la EN y la RSE son posibles. La nueva infraestructura de la economía de la informatización requiere -al interno- una ideología para movilzar, disciplinar y controlar a los individuos. Al externo, la economía de la informatización y el modelo de administración que a ésta corresponde se encuentran en un marco político y social donde la empresa prima y en donde la ideología de la cultura de la empresa es la pieza clave (Floris, 1996, 156).

- La ideología administrativa asume la forma de cientificidad de una manera doble: Por un lado, la empresa demuestra la aplicación científica de los conocimientos de las ciencias naturales y la economía. Según Floris (1996, 176) sobre este hecho real reposa la racionalidad científica de la idelogía administrativa. Luego, las otra fuentes proporcionadas por las nuevas

13 Existe otro aspecto de la adopción de la ideología: "la socialización de psique" (Castoriadis, 1975). Éste es un proceso de creación, asimilación, ruptura del mundo de significaciones históricas y socialmente instituidas en la creación del individuo. En el caso presente, se trataría de un momento posterior en esa recreación de la psique, el cual permite la reproducción social. 
teorías de la empresa y otras ciencias sociales como la política y la sociología vienen a crear la máscara de ciencia y filosofía que caracteriza la ideología. En este sentido, la aplicación de las tecnologías de la información y la comunicación en la empresa es un ejemplo conspicuo. Por eso Floris señala con justeza, que la nueva ideología administrativa eleva a un grado superior de desarrollo a las ideologías precedentes.

- En consecuencia, la ideología de la administración tiene una hoja doble: hacia al interior provoca la legitimación, la aceptación y el concenso de los hombres que ahí trabajan; en suma ella integra. Al exterior, difunde "el espiritu de la empresa". Ambos procesos son vehículados y controlados por la comunicación corporativa que diviene en un util estratégico para la administración.

- Por lo tanto, la ideología administrativa y el predominio de la empresa capitalista y del mercado mundial (Floris, 1996; Cuevas, 2003, 2011) aparecen como la forma "natural" y mejor dela organización social entre los hombres. Funcionar bajo los principios de la empresa devien en simbolo de excelencia y eficacia para todo tipo de organización. Y el mercado deviene de manera practica y simbolica, en el reproductor de la vida social-natural, olvidándose que no es el dinero ni la mercancías, ni la técnología, ni siquiera el conocimiento los que crean la vida social sino los hombres organizados dentro del modo de vida burgués (Marx, 1857, 1965a). De esta forma se consuma el proceso de mixtificación que caracteriza todo ideología.

En este sentido, D'Humières (2005) tiene razón al considerar que la idelogía de la empresa es una acumulación histórica de hechos y valores. Pero a diferencia, de Floris (1996) Landier (1991) Faber (1992), Marx y Engels (1846, 1982) en el discurso D'Humières -como para la mayor parte de los especialistas- la ideología es despojada del contenido de poder, autoridad, disciplina y legitimación; incluso ella desaparece quedando en su lugar quedan sus elementos simples: la cultura de la empresa, la marca, el logo, los códigos de conducta, los informes de responsabilidad social. Así, la comunicación corporativa se convierte en la pieza clave de la mutuación de la empresa. El un nuevo marco proporcionado por la RSE hace que la comunicación corporativa se desarrolle a partir de los ejes económico, social y del cuidado al medio ambiente (D'Humières, 2005, 243-279).

En esta nueva comunicación corporativa dos valores morales son centrales: La responsabilidad que estructura el discurso de la comunicación y la confianza que representa el punto clave para el valor de marca y el valor de imagen de la empresa, pues según D'Humières $(2005,267)$ la RSE es una respuesta a la crisis de confianza de la que gozan las empresas al inicio de este siglo. En su operacionalización estos valores devienen en parámetros de la eficacia de la comunicación coporativa: (1) Estableciendo el nivel de confianza de la marca/ principales públicos. (2) El radio de inversión en el tiempo/ valor de imagen en el tiempo. (3) Auditoria cualitativa de la función de comunicación.

En resumen, la ideología del capital para la empresa se enriquece enormemente durante las dos ultimas décadas de siglo pasado e inicios del presente. Esta riqueza al igual que su diversificación de la comunicación corporativa al externo de la empresa hacen que la categoría de ideología formulada por Marx \& Engels $(1846,1982)$ siga siendo válida al propio tiempo de prolongar su alcance con las nuevas funciones de la ideología y sus dimensiones estudiadas por los especialistas.

Esto conduce al otro componente de la segunda dimensión de la administración moral de la empresa y los negocios, Business Ethics o RSE, que es la doctrinaria. Una vez establecida la idelología del capital para la empresa ésta se presenta como común a los hombres que en ella trabajan y con la tendencia a la aceptación general de la sociedad. Esto es un verdadero proceso de reificación, es decir, la ideología del capital se presenta como una verdad evidente con vida propia y por lo tanto, con autonomía y poder frente a la masa; luego, sus conceptos, principios y jucios se presentan como ciertos y justos. Desde ahí la empresa como institución puede prescribir el sentido, la dirección y el cómo debe 
desarrollarse ella misma junto con el organismo social. Los especialistas como D'Humières (2005) tienen razón al decir que la empresa actual deja de ser una entidad cerrada sin contacto con el exterior. Al contrario, la empresa abre sus puertas para comunicarse con el público. Esto comienza con la publicidad del marketing durante los años 1980.

La emergencia del DS y la RSE provocan una divesificación en la comunicación de la empresa; luego a la filantropía de los años 1970 se suman durante las siguientes dos décadas las actvidades como el sponsoring (patrocinio), el mecenado, el deporte y la educación. En fin, a partir de 1990's-2000's se establecen los Informes de Responsabilidad Social o Desarrollo Sustentable.

Pero la comunicación, por trascendente que ésta sea, es un medio para vehicular y reproducir las relaciones entre los hombres. La comunicación y el lenguaje es una forma de inteligencia practica en la transformación del mundo. El hecho es que "al salir de la sombra" y mostrarse, la gran empresa predica cómo el mundo debe ser (Cuevas, 2003). Y lo hace asumiendo los valores morales, políticos y significados de la sociedad sobre la que ésta reposa. Decir que "...la empresa abre sus puertas para mostrarse..." es una mentira. Las empresas siguen siendo entidades cerradas con letreros tales "Prohibido el paso a toda persona ajena" "Admision solo por negocios". Para los administradores y propietarios, en general, no es grato recibir a un investigador "...que vea lo que está pasando ahí adentro." Lo que en realidad se quiere decir con la apertura de la empresa es que ésta muestra su papel activo en el espacio público mundial. La empresa deja de esperar que sea el Estado o los medios masivos sus voceros. Ella misma crea y difunde la conciencia que ella tiene de sí misma y cómo concibe la sociedad en el presente y en el futuro.

La noción de doctrina proviene del Latin (doctrina) que significa acción de enseñar, teoría y método. En la acepción que aquí interesa, la doctrina es un conjunto de principios los cuales pueden o no constituir un sistema. La doctrina establece una concepción del universo, de la existencia humana, de la sociedad o del mundo en general. Ésta se traduce en modelos de pensamiento y normas de conducta. En este sentido, según Lalande $(2002 ; 1926,244)$ la doctrina es también un conjunto de principios considerados como verdaderos, los cuales pueden conducir a la acción. Una doctrina como la del Socialismo o el DS pretenden la transformación del mundo y del comportamiento de los individuos. Pero mientras el socialismo pretende la transformación radical del organismo social, el DS busca una evolución sin cambiar el modo de vida capitalista.

Este hecho diferencia la política de las empresas capitalistas de épocas pasadas. Las empresas al asumir la doctrina del DS, las Iniciativas, los Principios y los útiles (Códigos Éticos, Auditorías, Índices) crean la RSE. Pero al hacerlo algunas son conducidas inexorablemente a la acción. Empresas como Alcatel, Danone, Renault, Philips, Nike al impulsar el cuidado del medio ambiente, la seguridad, la salud, la educación, el deporte y cumplir o no cumplir, los derechos universales de hombre, inciden sobre los seres humanos y la naturaleza de la región donde la empresa promueve esas actividades.

Los especialistas en DS y RSE tienen razón al subrayar ese cambio en la relación empresa y sociedad. Hasta antes del decenio 1990 son otras instituciones como el Estado, los Partidos Políticos, la Universidades, los Organismos Internacionales y los medios masivos de comunicación los encargados de difundir la ideología del capital y promover las transformaciones necesarias en el espacio público para el desarrollo del modo de vida capitalista y la empresa. Hoy en día, la ideología y la doctrina del DS y la RSE son promovidos por la empresa misma. Evidentemente, eso no quiere decir que los actores que antes difundían la ideología liberal hayan dejado de hacerlo. Al contrario, ahora más que nunca el Estado, los Partidos Políticos, la Universidades, los Organismos Internacionales y los medios masivos de comunicación continúan difundiendo la ideología del modo de vida burgués junto con la empresa misma. Estas instituciones trabajan bajo los valores morales de la sociedad burguesa así como bajo los principios metódicos y racionales de este modo de vida.

De igual modo, las empresas promueven la transformación del mundo -dentro del modo de vida bur- 
gués- junto con el Estado. Los especialistas consideran que eso se debe a una pérdida de hegemonía de este último la cual es aprovechada por la gran empresa capitalista para ser protagonista en el espacio público. En esa tesis subyace la idea de una contradicción entre el Estado y la Empresa. Contradicción del todo aparente pues ambos son producto del modo de vida burgués. El Estado burgués es, tanto más fuerte, como lo sea la empresa y la clase que éste representan.

El hecho importante es que la gran empresa capitalista afecta directamente la vida de la sociedad y el medio ambiente. Del mismo modo, este hecho es necesario ubicarlo en su justa medida. Los índices de DS y la RSE, las empresas adheridas a las Iniciativas de la ONU o la OCDE hablan de un número aún limitado de empresas en relación al universo de las mismas. Al contrario, el número de empresas que toman la vía del DS y la RSE puede ser pequeño en relación al universo, pero de envergadura, de acuerdo a su talla y capital que manejan. Sin embargo, el resultado sigue siendo exiguo en relación a la devastación del planeta Tierra. Así cuando la doctrina permanece en la inactividad, esta tiende ser ideología, primando sobre la dimensión de herramienta de la administración moral de la empresa y los negocios. Y una vez más, el modo de vida capitalista es la explicación de ese primado. Sin embargo, este hecho representa aún la oportunidad de una verdadera transformación moral dentro del marco de este modo de vida. Al parecer esto será posible solo cuando la catástrofe planetaria sea inminente.

\section{Resumen y conclusión}

En este artículo propone una guía de lectura para el estudio de la RSE mediante tres dimensiones: El conjunto de herramientas para su aplicación y evaluación constituye la dimensión de útil para la administración. Al discurso corresponde la dimensión ideológica y doctrinaria. Estas dimensiones permiten comprender el contenido y la forma de la RSE.

Esa triada de dimensiones supone la concepción de la empresa como una totalidad de relaciones económicas, políticas, sociales, simbólicas, éticas, institucionales y culturales en el contexto del modo de vida burgués. Eso supone que las dimensiones de la RSE es el resultado de la observación de la praxis realizada por los hombres en las empresas y las organizaciones; no se trata evidentemente, de una construcción a priori a la cual debe ajustarse el concepto como la de Carroll (1979) por ejemplo.

La RSE tiende a englobar los temas abordados por la EN y el DS (eficiencia económica, empleo, derechos del hombre, cuidado del medio ambiente, relaciones con los stakeholders, salud y cultura) por lo que se tiende hablar simplemente de RSE.

Con la aparición de la Ética de los Negocios (EN), comienzan a elaborarse una serie de herramientas para la administración moral de la empresa y los negocios. En una primera instancia estos útiles sirven para implementar la RSE, presentarla y finalmente intentar su evaluación. Los primeros de esos instrumentos son las Cartas y Códigos Éticos. Luego, con la institucionalización de la RSE aparecen durante la década de los noventas los Informes Sociales, y sus guías para implementarlos, por ejemplo, Global Reporting Initiative (1997) o ISO 26000 (2010). Paralelamente se construyen los índices de DS como el ASPI, Ethibel Excellence y Ethibel Pioneer y Dow Jones Sustainability Indexes, las Normas como las ISO 14000, AA 1000 (2003), SA 8000 , y las Auditorias Éticas. De igual modo las Iniciativas y Principios (i.e. Principios directores de la OCDE) constituyen verdaderas guías para la implementación de la RSE.

Al discurso corresponde la dimensión ideológica y doctrinaria; en su aplicación éstas se entrelazan en la praxis de la administración. La dimensión ideológica presenta la ideología administrativa o del capital como un discurso común al conjunto de organizaciones y la sociedad. Tomando la apariencia de ciencia o saber la ideología administrativa, usando la noción de Floris (1996) utiliza la comunicación corporativa para realizar los procesos de difusión aceptación y legitimación de esa ideología dentro de la empresa y en el espacio público.

Una vez establecido este proceso la Empresa propone estados deseables del mundo que conducen a los 
hombres a la acción de acuerdo a los valores morales y principios que ésta considera los pertinentes. Invadiendo este terreno la praxis de los hombres de la Empresa junto con las organizaciones crean la dimensión doctrinaria de la RSE.

En suma el proceso tridimensional descrito como una guía de lectura en el estudio de la RSE contrasta por un lado, con el efecto que la doctrina del DS y la RSE tiene sobre un verdadero mejoramiento en el bienestar, la justicia social, el respeto de los derechos universales y del medio ambiente. El modo de vida capitalista en donde las decisiones se determinan por el poder privado aparece como la piedra de toque de esa contradicción.

\section{Referencias}

Alchian, A-A \& Demsetz, H. (1972). Production, Information Costs, and Economic Organization. The American Economic Review, Vol. 62, No. 5 (Dec., 1972): 777-795.

Aupperle, K., A. B. Carroll \& J. D. Hatfield. (1985). An empirical examination of the relationship between corporate social responsibility and profitability. Academy of Management Joumal, 28(2): 446-463.

Bouilloud, J-P. \& Lecuyer, B-P. (1994). L'invention de la gestion. Histoire et pratiques, Paris Harmattan.

Callan, S. J.1 \& Thomas, J-M. (2009). Corporate financial performance and corporate social performance: an update and reinvestigation. Corporate Social Responsibility \& environmental Management; Mar/Apr2009, Vol. 16 Issue 2: 61-78, 18, 5 Charts.

Carroll, A. (1979). A three-dimensional conceptual model of corporate performance». Academy of Management. The Academy of Management Review, vol. 4 no 4: 497-506.

Castoriadis, C. (1975). L'institution imaginaire de la société, Éditions du Seuil, Paris.

Crifo \& Ponssard, (2008). RSE et/ou performance financière: points de repère et pistes de recherche Responsabilité, Ecole Polytechnique, Centre National de la Recherche Scientifique, Cahier n 2008-15, Août 2008.

CNUED (1992): Déclaraction de Rio Sur l'Environnement et le Développement. Rio de Janeiro, Brasil, 3-14 juin, ONU, Obtenido de: http://www.un.org/french/events/rio92/aconf 15126vol1f.htm.

CNUED. (1992). La Action 21, Nations Unies. Développement Durable, Cumbre de Río Obtenido de: http://www.un.org/ french/ga/special/sids/agenda21/action0.htm

Chauveau \& Rosé, (2003). L'Entreprise responsable : Responsabilité sociale - Ethique, Paris, Édtions d'Organisation.

Cuevas, R. (2003). L'éthique de l'entreprise capitaliste: Essai sur "L'éthique de l'entreprise" en France et l'idéologie. France, Université de Corse Pascal Paoli, Institut d'Administration des Entreprises (IAE), Thèse de Docteur en Sciences de Gestion, 8 avril 2003.
Cuevas, R. (2005). La construction de l'éthique de l'entreprise. Première Partie. Les formes à partir de l'adoption de l'éthique. Revista de Administración, Núm. 216, UNAM.

Cuevas, R. (2005). La construction de l'éthique de l'entreprise. Seconde Partie. Les formes à partir de l'adoption de l'économie par l'éthique de l'entreprise"'. Revista de Administración, Núm. 217, UNAM.

Cuevas, R. (2006). La ética de los negocios y la economía de la informatización, Revista de Administración, Núm. 219 UNAM.

Cuevas, R. (2007).Las formas de la ética de los negocios: la síntesis o las formas propiamente dichas. La doctrina social de la iglesia cristiana". Revista de Administración, Núm. 221 UNAM.

Cuevas, R. (2011). Ética y responsabilidad social de las empresas: La nueva cultura de la época de la globalización. Un enfoque dialéctico aparecer en editorial Miguel Ángel Porrúa

Cuevas, R. (2010). L'éthique est-elle vraiment rentable? Colloque CIRIEC Canada-ACFAS : Les pratiques de responsabilité sociale et environnementale des entreprises publiques et d'économie sociale et les défis de leur déploiement. Universidad de Montréal, 10/mayo/2010.

De la Bruslerie, H (coordonnateur) (1992). Éthique, Déontologie et Gestion de l'Entreprise, Paris, éditeur ECONOMICA collection Gestion.

D'Humières, P. (2005). Le développement durable, Paris, Éditions d'Organisation.

Donalson \& Dunfee (194), Toward a unified conception of business ethics: Integrative Social Contracts Theory, Academy of Management Review, Vol. 19 No. 2: 252-284.

Commission des Communautés Européennes (C.C.E.) (2001). Livre Vert. Promouvoir un cadre européen pour la responsabilité sociale des enterprises, Bruxelles.

Faber, E. (1992). Main basse sur la Cité. Ethique et entreprise, France, éditeur Hachette.

Frederick, R.E. (2001). La ética en los negocios. Aplicación a problemas específicos en las organizaciones de negocios. México, OXFORD, University Press, Traducción del inglés Carril Villarreal, M. del Pilar.

Gélinier, O. (1991). L'éthique des affaires. Halte à la dérive, Paris, Editions du Seuil.

Griffin, J.J. \& Mahon, J.F. (1997). "The Corporate Social Performance and Corporate Financial Performance Debate: Twenty-Five Years of Incomparable Research" Business and Society, Vol. 36, No. 1: 5-31.

Hardt, M. \& Negri, A. (2000). Empire, Paris Exils Editeur, traduit de l'américain par Canal, D-A

Hill, C. \& Jones, G. (2000). Administración estratégica un enfoque integrado, Colombia, McGraw-Hill.

Jones, T.M. (1980). «Corporate social responsibility revisited, redefined». California Management Review, vol. 22 no 3: 59-67.

ISO/DIS 26000 (2010). Lignes directrices relatives à la responsabilité sociétale — Guidance on social responsibility - Ginebra, Type du document: Norme internationale Sous-type du document: Stade du document: (40) Enquête, Langue du document: Française, International Organization for Standardization. 2009-10-08.

Kosik, K. (1970). La dialectique du concret, Traduit de l'allemand par Dangeville, R ; Paris, éditeur Maspero.

Koening, G. (coordinateur). (1999). De nouvelles théories pour gérer l'entreprise du XXI siècle, Paris. 
Landier, H. (1991). Vers l'entreprise intelligente. Dynamique du changement et mutation du changement, Paris, Editions Calmann-Lévy.

Lalande, A. (1991). Vocabulaire technique et Critique de la Philosophie, Paris, PUF, $199117^{e}$ édition.

Laville; E. (2006). L'entreprise verte, France, Village Mundial.

Lee, Min-Dong Pau. (2008). A review of the theories of corporate social responsibility: Its evolutionary path and the road ahead. International Journal of Management Review; Mar2008, Vol. 10 Issue 1: 53-73.

Leroux, A. (1995). Retour à l'idéologie. Pour un humanisme de la personne, Paris : PUF.

March, J-G. et Simon, H.-A; Les Organisations. Problèmes psychosocilogiques, Traduit para Rouchy J.-C. et Pruier, G. 2èm édition, 1999 (1958) Paris, Dunod.

Marom IY. (2006). Toward a unified theory of the CSP-CFP link". Journal of Business Ethics 67(2): 191-200.

Margolis, J. \& Walsh, J. (2003). Misery Loves Companies: Rethinking Social Initiatives by Business, ISSN, 0001-8392, Administrative Science Quarterly; Jun 2003, Vol. 48 Issue 2: 268$305,38,2$ charts.

Marx, K. (1857, 1965a). Introduction générale à la critique de l'économie politique, Paris, Bibliothèque de la Pléiade. CEuvres I Economie, édition établie, présentée et annotée par Maximilien Rubel. NRF Gallimard.

Marx, K. (1965b, 1867). Le Capital, livres I, II et III, Paris, Bibliothèque de la Pléiade. CEuvres I Economie, édition établie, présentée et annotée par Maximilien Rubel. NRF Gallimard.

Marx, K. \& Engels, F. (1982b, 1846). L'ldéologie allemande, Paris, Bibliothèque de la Pléiade. CEuvres III Philosophie, édition établie, présentée et annotée par Maximilien Rubel. NRF Gallimard.

McWilliams, A. \& Siegel, D. (2000). Corporate social responsibility and financial performance: correlation or misspecification?" Strategic Management Journal 21(5): 603-609.

Mercier, S. (1999). L'éthique dans les entreprises, Paris, La Découverte, Collection Repères.

Metivier, C. (2000). Cas pratique: Certification ISO 14001 d'un système de management environnemental = Practical case study on the implementation of ISO 14001 standard Presentado en el Séminaire international SFSTP No32, Montpellier, FRANCE (11/05/2000) 2000, vol. 10, no 5 («86 p.]) (bibl.: dissem.), pp. 306-307 tomado de http://cat.inist.fr/?aModele $=a$ fficheN\&cpsidt $=794969$

Moskowitz, M. (1972). Choosing socially responsible stocks, Business and Society Review, (1): 71-75.

Mintzberg, H. (1986; 1983). Le pouvoir dans les organisations, Paris, Les Editions de l'Organisation,

Arthur T. Denzau, A.T. \& North, D. (1994). Shared Mental Models: Ideologies and Institutions, Working Paper, Washington University St Louis \& Center for Politics and Economics Claremont Graduate School, 1994. obtenu de: http://econpapers. repec.org/RAS/pno11.htm

North, D. (1993). Five propositions about institutional change, Working Paper, Washington University St Louis, 1993. obtenu de: http://econpapers.repec.org/RAS/pno11.htm

North, D. (1994). Institutional change: A framework of analysis", Working Paper, Washington University St Louis, 1994. obtenu de: http://econpapers.repec.org/RAS/pno11.htm
North, D. (1996). Institutions, organizations and market competition, Working Paper, Washington University St Louis, 1996. obtenu de: http://econpapers.repec.org/RAS/pno11.htm

O'Bannon, D. P. \& L. E. Preston (1993). The corporate socialfinancial performance relationship: A typology and analysis, paper presented at the 1993 Annual Meeting of the Academy of Management, Atlanta, GA.

ONU. (2002). Rapport du Sommet mondial pour le développement durable Johannesburg, Afrique du Sud, 26 août-4 septembre 2002. Obtenido de: http://www.sommetjohannesburg.org/

ONU. (1992). Rapport de la Conférence des Nations Unies sur l'environnement et le développement, Rio de Janeiro, 3-14 juin 1992 (publication des Nations Unies, numéro de vente: F.93.I.8 et rectificatifs). Obtenido de: http://www.un.org/ french/events/wssd/pages/cnued.html

Orlitzky, M. Schmidt, F. \& Rynes, S. (2003). Corporate Social and Financial Performance: A Meta-analysis, Organization Studies (01708406); 2003, Vol. 24 Issue 3: 403-441, 39.

Persais E. (2003). Le rapport de développement durable (ou stakeholders' report): un outil pour une gouvernance sociétale de l'entreprise? Journée AIMS Développement durable et entreprise, 15 mai, Angers, 2003.

Peters, T. \& Waterman, R. (1983, 1982). Le prix de l'excellence. Les secrets des meilleures entreprises, Paris, InterEditions, Traduit de l'américain par Garene, M et Pommier, C.

Pesqueux, Y. \& Biefnot, Y. (2002). L'éthique des affaires. Management par les valeurs et responsabilité sociale, Paris, Éditions d'Organisation.

Porter, M. E. \& Kramer, M. R. (2002). The Competitive Advantage of Corporate Philanthropy Harvard Business Review; Dec2002, Vol. 80 Issue 12: 56-69.

Ramboarisata, L.N. (2010). Analyse institutionnelle de la stratégie de responsabilité sociale d'entreprise (RSE) des institutions financières coopératives, UQAM, ESG, Thèse, du Doctorat en Administration, sep/2009.

Sethi, P. (1975). Dimensions of corporate social performance: An analytic framework. California Management Review, vol. 17: 58-64.

Smith, A. (1999). Théorie des sentiments moraux, Traduction par Biziou, M, Gautier, C, Pradeau, J ; Paris, PUF.

Smith, A. (1991). Recherche sur la nature et les causes de la richesse des nations, Traduction de Garnier, G; Paris GF. Flammarion édition.

Solomon, R.C. (2000). Nuevas reflexiones acerca de las organizaciones de negocios. El éxito basado en la integridad de las personas, México, Oxford University Press, Traducido del inglés por María del Pilar Carril Villarreal.

Taylor, W. F. (1986, 1911). Los principios del management científico, España, Ediciones Orbis, S.A. Traducción Alicia Arrufat, quinta édition.

Vigeo (2010). Oferta de servicios de Auditoria catalogo electrónico,. Disponible en: http://www.vigeo.com/csr-rating-agency/ fr/offre-de-services-rse/audits-en-rse/la-gamme-overnance. html

Vogel, D. (2008, 2006). Le marché de la vertu. Possibilités et limites de la responsabilidad sociale des entreprises. Paris, ECONOMICA. Traduction de l'américain par Terny, J-G. 
Wartick, S. \& Philip C. (1985). The evolution of the corporate social performance model ». Academy of Management. The Academy of Management Review, vol. 10 no 4: 758-769.

Williamson, O.E. (1979). Transaction Cost Economics: The Governance of Contractual Relations, Jounal of Law and Ecomomic (No. 2, 1979).
Williamson, O.E. (1994). Les institutions de l'économie, Paris, InterEditions.

Wood, D. (1991). Corporate social performance revisited. Academy of Management. The Academy of Management Review, vol. 16 no 4: 691-718. 